\title{
Arbeitszeiten mobil Beschäftigter: Welche Auswirkungen haben auswärtige Übernachtungen?
}

\author{
Hiltraud GRZECH-SUKALO, Gerlinde VOGL ${ }^{1}$ \\ Universität Oldenburg
}

\begin{abstract}
Im vorliegenden Beitrag werden Ergebnisse von Arbeitszeiterhebungen bei mobiler Arbeit vorgestellt, die im Rahmen des Forschungsprojektes prentimo (Präventionsorientierte Gestaltung mobiler Arbeit) anhand von drei Unternehmen (in zwei Unternehmen durch qualitative Erhebungen, in einem durch die Analyse von konkreten Arbeitszeiten) analysiert wurden. Im Fokus steht dabei mobile Arbeit, die mit räumlicher Mobilität einhergeht. Mobilität ist zur Erledigung der Arbeitsaufgabe notwendig, wenn beispielsweise direkt beim Kunden gearbeitet wird. Zentrale Fragestellung des Beitrages ist, ob sich bei ÜbernachterInnen bzw. und NichtÜbernachterInnen unterschiedliche Auswirkungen hinsichtlich verschiedener Arbeitszeitmerkmale (Dauer der täglichen Arbeits- und Ruhezeiten, Verlässlichkeit des Arbeitsendes, Pausen, Wochenarbeitszeiten sowie Wochenendarbeit) ergeben. Als differenzierende Belastungsfaktoren zeigen sich überlange tägliche Arbeitszeiten, verkürzte Ruhezeiten, ein schwer kalkulierbares Arbeitsende, eine erhöhte Wochenarbeitszeit, Reisezeiten, Rufbereitschaften sowie eine unzureichende Routenplanung. Schlüsselwörter: mobile Arbeit, Arbeitszeit, Arbeitsende, Wochenarbeitszeit, Fahrtzeiten
\end{abstract}

\section{Arbeitszeit bei mobiler Arbeit: ein Problemaufriss}

Im Zeichen der Digitalisierung ändert sich die Arbeitswelt rasant. Ein markanter Aspekt dieser Veränderungen ist, dass Arbeit zunehmend mobil geleistet wird. Moderne, miteinander vernetzte Endgeräte wie Smartphones, Tablets und Laptops ermöglichen es, dass in immer mehr Bereichen mobil gearbeitet werden kann und wird.

$\mathrm{Zu}$ mobiler Arbeit liegen verschiedene Definitionen vor (vgl. u.a. Breisig et al. 2017; Ducki/Nguyen 2016; empirica 2000; Vogl et al. 2014), der gemeinsame Nenner liegt in der räum-

\footnotetext{
${ }^{1}$ Hiltraud Grzech-Sukalo ist wissenschaftliche Mitarbeiterin im Forschungsprojekt prentimo (Präventionsorientierte Gestaltung mobiler Arbeit) an der Carl-von-Ossietzky-Universität Oldenburg: hiltraud.grzech-sukalo@uni-oldenburg.de. Gerlinde Vogl ist wissenschaftliche Mitarbeiterin und Koordinatorin im Forschungsprojekt prentimo an der Carl-von-OssietzkyUniversität Oldenburg: gerlinde.vogl@uni-oldenburg.de.
} 
lichen und zeitlichen Flexibilität des Arbeitsortes sowie in der Nutzung von mobilen Endgeräten (vgl. Deutscher Bundestag 2017).

Immer mehr Orte werden so zu Arbeitsorten, dank der mobilen Technologien kann Reisezeit zugleich aktive Arbeitszeit sein, Transiträume wie Flughäfen, Bahnhöfe und Hotels können zu temporären Arbeitsorten, Verkehrsmittel zu rollenden Büros werden.

Örtliche und zeitliche Flexibilität kann für Beschäftigte Entlastung bringen, kann aber auch - bedingt durch hohe Mobilität, damit einhergehende Abwesenheitszeiten und permanente Erreichbarkeit - soziale und gesundheitliche Beeinträchtigungen nach sich ziehen (Beermann et al. 2017; Dettmers et al.; Ducki/Nguyen 2016; Hupffeld u.a. 2013).

Die Ortsveränderlichkeit von Arbeit bedingt oftmals auch räumliche Mobilität, die zur Erledigung der Arbeitsaufgabe erforderlich ist, wie dies z.B. bei Reisen zum Kunden oder zu Messen oder Tagungen der Fall ist. Diese Form mobiler Arbeit steht im Zentrum des Beitrags. Orts- und zeitflexibles Arbeiten ist hier nicht frei gewählt, sondern zur Erledigung der Arbeitsaufgabe eine schlichte Notwendigkeit. So müssen Servicetechniker und IT-Berater zum Kunden reisen, um dort eine Anlage zu warten, eine Reparatur vorzunehmen, oder einen neuen Auftrag zu akquirieren.

Mobilität bzw. Mobilsein benötigt Zeit und ist damit auch ein Zeitfresser (Vogl/Nies 2013; Vogl et al. 2014; Nies et al. 2017): Notwendige Reisezeit lässt sich nicht immer als produktive Arbeitszeit nutzen, nicht immer ist Reisezeit auch Arbeitszeit. Bei mehrtägigen Dienstreisen wird nicht nur beim Kunden, sondern auch noch anschließend im Hotel gearbeitet, um die anfallende Arbeit einerseits zeitnah zu erledigen und andererseits ebenfalls zeitnah wieder zuhause sein zu können. So

zeigt sich, dass örtliche Flexibilisierung zu erhöhten Arbeitszeiten beitragen kann. Dies ist in besonderem Maße dann der Fall, wenn die örtliche Flexibilisierung tätigkeitsimmanent ist und damit fremdbestimmt erfolgt. (Piele/Piele 2017: 8).

Zudem kann Reisezeit gerade bei Vertrauensarbeitszeit „verschwinden“, sie gilt nur bedingt als Arbeitszeit und wird deshalb auch nur anteilig zur Arbeitszeit gerechnet. Das UnterwegsSein bindet dadurch sowohl berufliche als auch private Zeit und kann zu einer erheblichen Ausdehnung „realer“ Arbeitszeit und zu Konflikten mit der Work-Life-Balance führen (Pangert et al. 2016; Pangert/Schüpbach 2014).

Die Dokumentation und damit auch die Auswertung von Arbeitszeiten bei mobiler Arbeit steht vor diversen Herausforderungen: Arbeitszeit, die außerhalb des Kontrollbereichs des Betriebs erbracht wird, wird nicht oder nur zum Teil erfasst. Zudem werden Arbeitszeiterfassungen sehr selten für externe Auswertungen zur Verfügung gestellt. Bedenken bestehen bzgl. der Aufdeckung möglicher Verstöße gegen das Arbeitszeitgesetz (2003). Von daher verwundert es nicht, dass es bislang kaum belastbare Daten bzw. Auswertungen zu den tatsächlich geleisteten Arbeitszeiten und deren Abfolge bei mobiler Arbeit gibt.

Neben der Betrachtung einzelner Arbeitszeitmerkmale sollten diese Merkmale, wenn möglich, in Kombination und Abfolge über einen längeren Zeitraum analysiert werden. Benötigt werden hierfür die geleisteten Arbeitszeiten z.B. in Form von Arbeitszeitdokumentationen. Diese zur Verfügung zu stellen, erweist sich für viele Unternehmen als Herausforderung, 
die sie nicht selten scheuen. Dies liegt u.a. daran, dass potentielle gesetzliche Verstöße in den Arbeitszeiten deutlich ersichtlich sein können.

Mobile Arbeit kann durch betriebliche Vorgaben und Planungen der Arbeitseinsätze, durch Vorgaben der Kunden sowie durch längere Abwesenheitszeiten zu Belastungen führen. So zeigt sich, dass sich bei mobiler Arbeit Auswirkungen auf die Einhaltung von Ruhe- und Pausenzeiten sowie auf tägliche oder wöchentliche Arbeitszeiten, ergeben (vgl. Grzech-Sukalo 2017; Grzech-Sukalo et al. 2017). Ein wichtiger Aspekt ist dabei, ob sich bei mehrtägigen oder wochenlangen Abwesenheitszeiten durch auswärtiges Übernachten zusätzliche Belastungen hinsichtlich kritischer Arbeitszeitmerkmale ergeben.

Der Analyse der Arbeitszeiten mobil Beschäftigter liegt im Hinblick auf Arbeitszeitmerkmale das Arbeitszeitgesetz zugrunde (ArbZG 2003). Gleichzeitig kann auf Erkenntnisse flexibler bzw. variabler Arbeitszeit und der Schichtarbeit zurückgegriffen werden (vgl. u.a. Janßen/Nachreiner 2004; Wirtz 2010; DGUV 2012; Nachreiner 2017). Aus diesen Untersuchungen ist schon länger bekannt, dass tägliche Arbeit dauerhaft über acht Stunden oder Wochenarbeitszeiten spätestens ab 45 Stunden, je nach Arbeitsbelastung auch schon wesentlich früher, zu psychischen und physischen Beeinträchtigungen führen können.

Bei täglichen Ruhezeiten unter dem gesetzlich vorgeschriebenen Mindestwert von 11 Stunden zeigt sich, dass die Leistungsfähigkeit der Beschäftigten sinkt und die Gefahr von Fehlern und Unfällen steigt (DGUV 2012; Nachreiner 2017). Zudem haben sich nicht vorhersehbare und damit nicht planbare Arbeitszeiten immer wieder als ungünstig herausgestellt hat, insbesondere hinsichtlich des Schlafs sowie anderer körperlicher und sozialer Aspekte.

Auch Wochenendarbeit kann sich, gerade wenn sie nicht nur vereinzelt und planbar vorkommt, negativ auf die Work-Life-Balance auswirken und zu sozialen, aber auch gesundheitlichen Beeinträchtigungen führen.

Die Gefährdung durch lange tägliche Arbeitszeiten, hohe bzw. stark schwankende Wochenarbeitszeiten sowie zusätzlich durch reduzierte oder sogar fehlende Pausen kann sich noch verstärken, wenn eine Unkalkulierbarkeit der Arbeitszeit dazukommt. Dazu gehören z.B. ein unklares Arbeitsende oder auch Zeiten auf Abruf, wie sie durch Rufbereitschaften, gerade wenn diese vertraglich nicht ausreichend festgelegt werden (vgl. Böker/Demuth 2015), gegeben sind. Bei mobiler Arbeit ist dies zu vermuten, besonders wenn betriebliche Regelungen wie Vertrauensarbeitszeit vorliegen (Piele/Piele 2017).

Auch Reise- bzw. Fahrtzeiten, ${ }^{2}$ die zur Erledigung der Arbeitsaufträge notwendig sind, stellen beachtenswerte Aspekte dar. Dabei deutet sich an, dass hierfür einerseits eine Fehlplanung der Arbeitseinsätze verantwortlich sein kann, wenn z.B. die zur Erledigung der Arbeitsaufträge zugrunde gelegten Zeiten für die Einsätze selbst, die benötigten Fahrzeiten zu Kunden oder auch Zeiten für Parkplatzsuche zu gering bemessen werden. Andererseits kann hierfür auch eine zu geringe Personalbedarfsbemessung verantwortlich sein. Bedeutsam sind in diesem Zusammenhang auch die vertraglichen Regelungen in den Unternehmen im Hinblick auf die Anrechnung von Fahrtzeiten als Arbeitszeiten.

\footnotetext{
${ }^{2}$ Fahrt- oder Reisezeiten als auch Geschäftsreisen werden oftmals synonym verwendet. Eine klare Unterscheidung findet sich bei Gerstenberg et al. (2017). Im Projekt prentimo und damit in der hier vorliegenden Betrachtung liegt der Fokus auf Arbeits- und Reisezeiten, die im Zusammenhang mit der Ausübung der beruflichen Tätigkeit anfallen, d.h. dass die Wege zur Arbeit, also das Pendeln, nicht berücksichtigt wird.
} 
Ein wichtiger Unterschied bei den mobil Beschäftigten der betrachteten Unternehmen ist, ob es sich um so genannte ÜbernachterInnen oder Nicht-ÜbernachterInnen handelt (vgl. Schneider et al. 2016). Nicht-ÜbernachterInnen sind in der Regel abends wieder zuhause, während ÜbernachterInnen zumeist mehrere Tage in der Woche oder auch über längere Zeiträume vor Ort beim Kunden sind und in Hotels übernachten. Die Unterscheidung zwischen ÜbernachterInnen und Nicht-ÜbernachterInnen ist insbesondere hinsichtlich der Auswirkungen auf Familie und Partnerschaft relevant, kann aber auch Auswirkungen auf die Arbeitszeiten nach sich ziehen, wie im Folgenden gezeigt wird.

\section{Das Forschungsprojekt prentimo}

Im Rahmen des Forschungsprojektes prentimo ${ }^{3}$ (Präventionsorientierte Gestaltung mobiler Arbeit) arbeiten wissenschaftliche und gewerkschaftliche Institutionen mit Unternehmen zusammen, um durch betriebliche Gestaltungsmaßnahmen mobile Arbeit nachhaltig gesund zu gestalten. Dazu wurden zunächst die konkreten Arbeitssituationen und -bedingungen erfasst, wozu auch Merkmale von Arbeitszeiten gehören, wie tägliche Arbeits- und Ruhezeiten, Pausen oder Wochenendarbeit. Basierend auf den Ergebnissen wurden und werden in den beteiligten Unternehmen betriebliche Gestaltungsprojekte und Interventionen zu mobiler Arbeit angeregt, begleitet und gestaltet.

\section{Der Methodenmix}

Zum Einsatz kam ein Methodenmix aus qualitativen und quantitativen Erhebungsinstrumenten. Insgesamt wurden 38 qualitative Interviews (s. Tabelle 1) sowie 6 ExpertInneninterviews von 4 Interviewerinnen durchgeführt, die vorab hinsichtlich der Inhalte und der Durchführung trainiert wurden. Als Experten zu betrieblichen Rahmenbedingungen und Praktiken wurden in den beteiligten Unternehmen Personalverantwortliche und -entwickler, Arbeitsschützer, Gesundheitsbeauftragte sowie Mitglieder der Interessenvertretung zu den betrieblichen Rahmenbedingungen befragt.

In den 60 bis 90 Minuten dauernden leitfadengestützten Intensivinterviews mit mobil Beschäftigten standen - basierend auf den betrieblichen Regelungen - die subjektiven Mobilitätspraktiken im Zentrum. Die Interviewführung orientierte sich dabei am verstehenden Interview nach Kaufmann (1999), der großen Wert auf die Offenheit in der Interviewsituation legt. Der Interviewleitfaden diente folglich als Wegweiser und nicht als starres Konzept für die Gespräche, so dass die Interviewten im Gespräch auch eigene Schwerpunkte setzen konnten. Die Interviews wurden vollständig transkribiert und im Anschluss einem interpretativ und typisierend angelegten Auswertungsverfahren unterzogen (zur Vorgehensweise s. Menz/Kratzer 2015).

\footnotetext{
${ }^{3}$ Das Vorhaben wird durch das Bundesministerium für Bildung und Forschung im Rahmen des Programms „Arbeiten Lernen - Kompetenzen entwickeln. Innovationsfähigkeit in einer modernen Arbeitswelt“ im Förderschwerpunkt „Präventive Maßnahmen für die sichere und gesunde Arbeit von morgen“ gefördert. Laufzeit des Projektes: 01.01.2016 bis 30.04.2019. Nähere Informationen zum Projekt finden sich unter: www.prentimo.de
} 
In fünf Unternehmen wurden Mitarbeiterbefragungen zu den Bedingungen mobiler Arbeit durchgeführt (vgl. dazu Kraus/Rieder 2018). Hinsichtlich der Arbeitszeiten kamen ausgewählte Fragen des 6th EWCS (European Working Condition Survey, Eurofound 2015) zur Anwendung.

\section{Die Stichprobe}

In prentimo sind insgesamt sechs Betriebe eingebunden, die sich durch eine hohe Heterogenität hinsichtlich Branche und Unternehmensgröße auszeichnen. Die Stichprobe ist nicht repräsentativ, die Rahmenbedingungen hinsichtlich Mobilität (insb. bei ÜbernachterInnen bzw. Nicht-ÜbernachterInnen) aber durchaus vergleichbar.

Die folgenden Ausführungen beziehen sich auf drei Unternehmen, in denen spezielle Erhebungen und Auswertungen zur Arbeitszeit stattgefunden haben. ${ }^{4}$ Konkrete Arbeitszeitaufzeichnungen über einen längeren Zeitraum liegen allerdings nur bei Unternehmen 2 vor. Unternehmen 1: IT-BeraterInnen und VertrieblerInnen eines Beratungsunternehmens. Unternehmen 2: Ingenieure und Techniker eines Anlagenbauers.

Unternehmen 3: Service- und WartungstechnikerInnen eines Telekommunikationsunternehmens.

Während die Service- und WartungstechnikerInnen (Unternehmen 3) am Tag mehrere Kundentermine in einem begrenzten geografischen Raum abarbeiten und abends wieder zuhause sind, legen die IT-BeraterInnen und VertrieblerInnen (Unternehmen 1) sowie die Ingenieure und Techniker im Anlagenbau (Unternehmen 2) weitere Strecken zurück, meist verbunden mit auswärtigen Übernachtungen.

In der nachfolgenden Tabelle 1 werden die drei Unternehmen vorgestellt und hinsichtlich der Mobilität, der verschiedenen Arbeitszeitregelungen sowie der eingesetzten Methoden beschrieben.

Bei Unternehmen 1 sind die mobil Beschäftigten überwiegend national, teilweise auch international unterwegs. Sie können zwischen Gleitzeit oder Vertrauensarbeitszeit mit Zielvereinbarung und individuell ausgehandelten Gehältern wählen, wobei Vertrauensarbeitszeit bevorzugt wird. Die Fahrten zu den Kunden erledigen die Beschäftigten überwiegend mit dem Auto. Auswärtiges Übernachten vor Ort bei den Kunden, auch mehrere Tage in der Woche, gehört zum Arbeitsalltag.

Unternehmen 2 ist ein global agierender Anlagenbauer, der Einzelteile zu kompletten Anlagesystemen montiert, installiert und programmiert. Hierbei handelt es sich u. a. um nichtvollautomatische Qualitätsprüf- und Montageplätze inkl. Robotik und Kamerasystemen. Mobil Arbeitende sind Ingenieure und Techniker, ${ }^{5}$ welche für die Programmierung der Software sowie für den Aufbau und die Inbetriebnahme der Geräte und Arbeitsstationen zuständig sind. Die mobilen Ingenieure und Techniker sind national und international unterwegs. Die Anreise zu den Kunden erfolgt, je nach Entfernung, mit Auto, Bahn oder Flugzeug. Fahrt-

\footnotetext{
${ }^{4}$ Die Arbeitszeiten von mobil Beschäftigten wurden nicht in allen Unternehmen systematisch erhoben oder nicht für externe Auswertungen zur Verfügung gestellt.

${ }^{5}$ Die männliche Schreibweise wurde gewählt, da die Stichprobe bei Unternehmen 2 nur Männer umfasst.
} 
bzw. Reisezeiten werden als Arbeitszeiten angerechnet. Übernachtungen vor Ort bei Kunden, auch über einen längeren Zeitraum, sind üblich.

Tabelle 1: Berücksichtigte Unternehmen, betriebliche Regelungen und verwendete Methoden

\begin{tabular}{|l|}
\hline ÜbernachterInnen \\
\hline Unternehmen 1: IT-Berater, VertriebsmitarbeiterInnen \\
Gleitzeit oder Vertrauensarbeitszeit mit Zielvereinbarungen \\
Anrechnung von Fahrtzeiten zur Arbeitszeit differiert, bei Vertrauensarbeitszeit geringer Anteil \\
Interviews mit 6 betrieblichen ExpertInnen (2 Frauen, 4 Männer) sowie mit 14 mobil Beschäftigten (3 Frau- \\
en, 11 Männer) \\
\hline \begin{tabular}{l} 
Unternehmen 2: Techniker und Ingenieure \\
Gleitzeit mit Rufbereitschaft \\
Fahrtzeit ist Arbeitszeit \\
Schriftliche Befragung von 40 Beschäftigten, 18 davon mobil tätig (keine Frauen) \\
\hline Nicht-ÜbernachterInnen \\
Unternehmen 3: Service- bzw. Wartungstechniker \\
Gleitzeit mit Rufbereitschaft \\
Fahrtzeit ist Arbeitszeit
\end{tabular} \\
Interviews mit 18 mobil Beschäftigten (4 Frauen, 14 Männer)
\end{tabular}

Mobile Arbeit kann hier sehr unterschiedliche Formen aufweisen: Von zweiwöchigen Einsätzen mit teils direktem Übergang zum nächsten Projekt bis zu mehrmonatigen oder mehrjährigen Einsätzen in der europäischen Zweigstelle als Tandem-Variante. Die Mitarbeiter sind an den Wochenenden nicht unbedingt zu Hause. Gemäß betriebsinterner Reiserichtlinie sind bis zu drei Wochen vor Ort beim Kunden zulässig. Die Planbarkeit der Einsätze ist ebenfalls unterschiedlich. Es kann vorkommen, dass der Einsatz eine Woche im Voraus noch nicht bekannt ist. Eine Vollzeitbeschäftigung umfasst 38,5 Stunden pro Woche.

Arbeitszeiten werden im Unternehmen generell elektronisch erfasst, vorrangig über den Laptop und das Handy. Es gilt eine Gleitzeitregelung mit einer Eingleitzeit von 07.00 bis 08.00 Uhr sowie Rufbereitschaften. Ob Arbeit, die im Hotel oder am Wochenende von den Beschäftigten geleistet wird, auch als Arbeitszeit verbucht wird, bleibt unklar. Die in Unternehmen 2 von mobil Beschäftigten tatsächlich geleisteten Arbeitszeiten in Dauer, Lage und Verteilung bilden einen wesentlichen Punkt im vorliegenden Beitrag.

In Unternehmen 3 liegt Gleitzeit mit Rufbereitschaft vor. Fahrten zum Kunden sind eher kurz und erfolgen mit dem Auto, die Zeiten gehören zur Arbeitszeit. Auswärtige Übernachtungen kommen in der Regel nicht vor.

In der betrachteten Stichprobe der drei Unternehmen sind Männer deutlich überrepräsentiert, im Unternehmen 2 gibt es keine mobil arbeitenden Frauen. Grund hierfür sind vorran- 
gig die Berufe der mobil Beschäftigten wie Anlagen- und Werkzeugmaschinenbauer, die überwiegend von Männern ausgeübt werden.

Im Folgenden wird der Fokus auf die Unterscheidung zwischen ÜbernachterInnen und Nicht-ÜbernachterInnen gerichtet und deren Arbeitszeiten im Hinblick auf die Dauer der täglichen Arbeits- und Ruhezeiten sowie auf das Arbeitsende, die Wochenarbeitszeit, Fahrtzeiten und Rufbereitschaften betrachtet.

Neben Ergebnissen aus den qualitativen Beschäftigteninterviews fließen auch erfasste Arbeitszeiten des Unternehmens 2 in die Analysen ein. Auch die vertraglichen Regelungen zur Arbeitszeit haben einen wesentlichen Einfluss. Die dargestellten Ergebnisse basieren auf den Erhebungen der in Tabelle 1 dargestellten drei Unternehmen.

\section{Ergebnisse der Auswertungen der qualitativen Beschäftigteninterviews}

Über erste Ergebnisse zu Arbeitszeit mobil Beschäftigter, die im Rahmen von prentimo erhoben wurden, wurde schon berichtet (Grzech-Sukalo 2017; Grzech-Sukalo et al. 2017). Folgende Merkmale haben sich hinsichtlich der Arbeitszeit von mobil Beschäftigten als relevant herausgestellt:

- die Dauer der täglichen Arbeitszeit

- das Arbeitsende

- die Wochenarbeitszeit

- Fahrtzeiten

- Rufbereitschaften

\section{Dauer der täglichen Arbeitszeit und Arbeitsende}

Mobilität an sich wird von den meisten Beschäftigten als positiv gesehen: „Das Unterwegssein finde ich total cool. Dieses Selbstbestimmte. Nicht von 8 bis 17 Uhr arbeiten zu müssen." (UN 1, MA 02). Negative Auswirkungen wie lange Arbeitszeiten oder ein schwer planbares Arbeitsende wurden erst im Laufe der Interviews genannt, da die Beschäftigten davon ausgehen, dass diese zu ihrem Beruf einfach dazugehören. Gerade Nicht-ÜbernachterInnen (s. Unternehmen 3) verfolgen vorrangig das Ziel, die vorgegebenen Kundenaufträge abzuarbeiten: „Ich sage immer, mein Feierabend richtet sich immer nach meinem letzten Auftrag." (UN 3, MA 03). Ein nicht kalkulierbares Arbeitsende führt dazu, dass private und familiäre Vereinbarungen weniger eingehalten werden können. Dies setzt von Seiten der Partnerinnen und Partner mobil Beschäftigter ein großes Verständnis voraus:

Arbeitsende, da gibt es im Grunde keine Definition. Meine Frau hat sich mittlerweile abgewöhnt mal anzurufen und zu fragen, wann bist Du denn zuhause? Das wissen wir nie (UN 3, MA 03).

Diese Stunden von Mehrarbeit werden nicht grundsätzlich als solche erfasst. Gleichzeitig wird ein unkalkulierbares Arbeitsende nicht durchgängig als negativ angesehen, sondern von Seiten der Interviewten besteht oft ein Eigeninteresse, die Arbeit bei den Kunden mit guter Arbeitsqualität zu beenden. 
Zudem ist die zeitliche Planung von Aufträgen teilweise zu knapp bemessen, was ebenfalls einen entscheidenden Einfluss auf das Arbeitsende hat und als belastend gesehen wird.

Die Zeiten, die da hinterlegt sind, sind einfach sehr, sehr knapp. [...] Aber diese kurze Zeittaktung, das finde ich als sehr belastend, weil man da keinen Einfluss drauf hat (UN 3, MA 10).

Arbeiten nach dem vertraglich geregelten Feierabend ist bei mobil Beschäftigten, sowohl bei ÜbernachterInnen als auch bei Nicht-ÜbernachterInnen, vorzufinden, wenn auch aus unterschiedlichen Gründen. So ist es gerade bei den Letztgenannten nicht ungewöhnlich, dass zuhause Mails abgefragt und beantwortet werden, dies auch nach 22.00 Uhr. Als Grund dafür wird die damit verbundene bessere Planung des nächsten Arbeitstages genannt. Zudem erfolgt nach Feierabend ein Austausch zwischen Kolleginnen und Kollegen, der durch fehlenden Kontakt tagsüber ansonsten nicht mehr möglich ist.

Bei ÜbernachterInnen wird aus anderen Gründen nicht selten länger als zehn oder auch zwölf Stunden gearbeitet. Als Erklärung hierfür werden u.a. der Mangel an anderen Beschäftigungen sowie das Fehlen von Freunden und Familie vor Ort genannt: „Und ich laufe durch irgendeine fremde Stadt, in der ich vielleicht abends gar nicht sein will“ (UN 1, MA 02).

\section{Wochenarbeitszeit}

Entstandene Mehrarbeit durch verlängerte tägliche Arbeitszeiten können NichtÜbernachterInnen durch tarifliche und betriebliche Regelungen meist zeitnah in Freizeit ausgleichen. Die vertraglich festgelegte Wochenarbeitszeit wird selten überschritten. Wochenarbeitszeiten auch über den gesetzlichen Rahmen hinaus sind eher bei ÜbernachterInnen (Unternehmen 1 und 2) vorzufinden, da meist geregelte Ausgleichsmöglichkeiten fehlen.

Also man arbeitet als Berater halt keine 40 Stunden. Das ist halt immer irgendwo mehr. Das gehört halt zum Geschäft. Wir haben eben Vertrauensarbeitszeit. [...] Mehraufwand wird erwartet, also es ist latent die Erwartungshaltung (UN 1, MA 05).

Die Schwankungsbreite für Mehrarbeit bewegt sich nach Angaben der Interviewten monatlich zwischen fünf oder sechs Stunden bis hin zu zehn Stunden innerhalb von 14 Tagen. Auch die Arbeit im Homeoffice, die nach Absprache möglich ist, zieht eine lange Erreichbarkeit nach sich, obwohl die Arbeitszeit mitbestimmt werden kann. Es ist nicht unüblich, zwölf Stunden erreichbar zu sein, zumeist zwischen 8.00 und 20.00 Uhr. Das Argument Selber schuld z.B. an langen täglichen Arbeitszeiten, wird angeführt um zu verdeutlichen, dass man eigentlich nicht dazu gezwungen wird, man könnte das Smartphone ja auch ausschalten.

Gibt es besondere Anfragen nach $20.00 \mathrm{Uhr}$, z.B. von KollegInnen, werden diese aus Gründen der Kollegialität auch noch bearbeitet. Gleichzeitig wird es als angenehm geschildert, im häuslichen Umfeld zu sein. Zudem wird, wenn möglich, nicht durchgehend gearbeitet, sondern es werden eigenverantwortlich Pausen genommen.

In den Interviews werden von ÜbernachterInnen Wochenarbeitszeiten von 48 Stunden und mehr als nicht unüblich genannt. Grundsätzlich wird 10 bis $20 \%$ Mehrarbeit geleistet, die nicht in die Berechnungen der Arbeitszeiten einfließt. Bei dem vorliegenden Unternehmen 
mit Vertrauensarbeitszeit werden darüber hinaus die Fahrtzeiten (s.u.) nur anteilig zu diesen Arbeitszeiten gerechnet in Abhängigkeit davon, ob sie den Kunden in Rechnung gestellt werden können oder nicht.

\section{Fahrtzeiten}

Fahrtzeiten, die zur Erledigung der Arbeit beim Kunden notwendig sind, stellen oftmals ein Problem dar. Einerseits, weil sie nicht immer zur Arbeitszeit gerechnet werden, was vorrangig für Unternehmen 1 und dort für die Beschäftigten in Vertrauensarbeitszeit gilt: „Ich verbringe dann einen halben Arbeitstag auf der Autobahn und trotzdem muss ich ja meinen vollen Arbeitstag da hinten schaffen" (UN 1, MA 02). Andererseits finden Fahrtzeiten auch in der Gesamtplanung der Kundenbesuche eine zu geringe Berücksichtigung. Vor allem Staus und langwierige Parkplatzsuche fließen nicht immer ausreichend in die Planung von Zeiten ein, die für den jeweiligen Auftrag veranschlagt werden. Dadurch ergibt sich ein weiteres Problem, das sich vorrangig bei den Nicht-ÜbernachterInnen findet. Die Zeit, die für jeden einzelnen Auftrag geplant wird, wird zunehmend knapper angesetzt. Dadurch steigt der zeitliche Stressfaktor (Zeitdruck) stark an, was von den Interviewten als Belastung benannt wird. In gleichem Maße wird nicht selten auf Pausen verzichtet, um die Arbeit bei den Kunden erledigen zu können.

Gerade bei ÜbernachterInnen erfolgt die Anreise zum Kunden - insbesondere dann, wenn die Anreise mit einer langen Flugreise verbunden ist - bereits am Sonntagabend, damit man am Montagmorgen ausgeschlafen beim Kunden ist. Durch die Reiseplanung wird der Sonntag nicht mehr als komplett arbeitsfrei erfahren und Auswirkungen auf das private Leben als auch auf den Schlaf, der kürzer und schlechter ausfällt, können die Folge sein:

Montagmorgen um 4 geht der Wecker. Da muss ich dann sonntags schon Koffer packen. Dann hat der Sonntag für mich eine ganze Menge an Erholung verloren [...] das Ganze zieht sich dann über die Woche, der mangelnde Schlaf, dann schläft man eh nicht so gut wie zuhause, dann bin ich am Freitag total k.o. (UN 1, MA 11).

\section{Rufbereitschaft}

Da mobile Arbeit stark mit flexiblen Arbeitszeiten verknüpft ist, ist Rufbereitschaft häufig vorzufinden, um Kundenwünschen ggf. rund um die Uhr gerecht werden zu können. Es ist ein wesentlicher Faktor, ob es sich um geplante oder ungeplante Rufbereitschaften handelt. Ungeplant können sich negative Auswirkungen auf das Privatleben und die Gesundheit ergeben: „Sie sitzen zu Hause, plötzlich klingelt das Telefon, der Teamleiter ist dran und sagt, ich habe noch eine wichtige Störung. Dann muss ich eben nochmal los“ (UN 3, MA 09). Rufbereitschaften, auch wenn diese freiwillig zu leisten sind, führen dazu, dass die betroffenen Beschäftigten wegen des Gefühls, permanent erreichbar sein zu müssen, diese als belastend empfinden. 


\section{Ergebnisse der quantitativen Auswertungen: Arbeitszeiten mobil Beschäftigter am Beispiel von Unternehmen 2}

Unternehmen 2 hat Auszüge aus dokumentierten Arbeitszeiten von 8 Beschäftigten, jeweils über 4 Monate, zur Analyse zur Verfügung gestellt. Dies und auch die vorgefundenen Ergebnisse sprechen dafür, dass es sich bei diesem Unternehmen um einen Betrieb handelt, der ein Interesse dran hat, die Arbeitszeiten und deren Grenzwerte nach dem Arbeitszeitgesetz einzuhalten. Zudem sind die vorliegenden Arbeitszeiten im Rahmen der Analyse mobiler Arbeit sehr hilfreich, da eine solche Dokumentation der Abfolge nach Lage, Dauer und Verteilung bis dato in der Literatur nicht vorzufinden ist. Umso wichtiger ist es, diese zu analysieren, wohl wissend, dass es sich bei diesem Beispiel um eine Positivauswahl mit kleiner Stichprobe handelt.

Nicht gesondert ausgewiesen werden in den Aufzeichnungen die Fahrtzeiten sowie Einsätze durch Rufbereitschaften, da beides zu 100\% in die Arbeitszeiten einfließt. Gleichzeitig ist nicht zu erkennen, wo sich die Beschäftigten befinden, ob sie also beim Kunden vor Ort bleiben oder nach Hause fahren können.

Wie schon erläutert, sind im Unternehmen 2 mobil Beschäftigte vorzufinden, die sehr häufig vor Ort beim Kunden arbeiten und während des Auftrags dort übernachten.

Tabelle 2 verdeutlicht die zur Verfügung gestellten tatsächlichen Arbeitszeiten von 8 mobil Beschäftigten im Überblick. Zudem gehen die Fragen zur Arbeitszeit mobil Beschäftigter aus der Mitarbeiterbefragung in die Tabelle ein. ${ }^{6}$ Da es sich lediglich um insgesamt 18 mobil Beschäftigte handelt, werden keine Prozentwerte aufgeführt, sondern absolute Werte.

Betrachtet man die vorgefundenen Arbeitszeiten, ergeben sich verschiedene Auffälligkeiten. So liegen die täglichen Arbeitszeiten nicht selten über 10 Stunden: 13 Beschäftigte geben an, 1-5-mal pro Monat so zu arbeiten, 2 Beschäftigte arbeiten monatlich sogar 6-10-mal über 10 Stunden. Die betrachteten Arbeitszeitaufzeichnungen zeigen bei allen betrachteten $8 \mathrm{Be}-$ schäftigten Verstöße (7-17-mal in 4 Monaten). Dies überschreitet eindeutig den gesetzlichen Rahmen. Zudem häufen sich diese langen täglichen Arbeitszeiten besonders, wenn vor Ort bei Kunden gearbeitet wird, was sich aus den Gesprächen mit der Geschäftsleitung ergeben hat. Die Dauer der täglichen Arbeitszeit kann bei langen Kundeneinsätzen z.T. über mehrere Wochen sehr hoch sein.

Dies führt einerseits zu reduzierten täglichen Ruhezeiten, wenn auch zumeist nur geringfügig, allerdings in Einzelfällen erheblich. Immerhin geben 13 Beschäftigte an, innerhalb eines Monats zumindest einmal weniger als die gesetzlich vorgeschriebenen mind. 11 Stunden tägliche Ruhezeit zu haben. In den Arbeitszeitaufzeichnungen lassen sich bei 5 der 8 betrachteten Beschäftigten Unterschreitungen der täglichen Mindestruhezeiten 1-4-mal im betrachteten Zeitraum vorfinden,

Andererseits ergeben sich hohe Wochenarbeitszeiten, da die tägliche Arbeitszeit bei Kunden vor Ort, wie sich aus den Diskussionen ergab, über den vom Gesetz vorgegebenen durchschnittlich 8 Stunden liegen. Damit kommt es zu stark schwankenden Wochenarbeitszeiten.

\footnotetext{
${ }^{6}$ Auszug basierend auf 6th EWCS, Eurofound 2015.
} 
Diese liegen bei 6 der 8 betrachteten Beschäftigten im analysierten Zeitraum 1-4-mal über 48 Stunden, wenn auch im gesetzlichen Rahmen.

Tabelle 2: Auswertung Arbeitszeiten im Überblick sowie dazugehörige Befragungsergebnisse (Unternehmen 2)

\begin{tabular}{|c|c|c|c|}
\hline Auswertung & $\begin{array}{l}\text { gesetzliche } \\
\text { Grenzwerte }\end{array}$ & $\begin{array}{l}\text { Arbeitszeitaufzeichnungen } \\
\text { von } 8 \text { Beschäftigten jeweils über } 4 \\
\text { Monate }\end{array}$ & $\begin{array}{l}\text { Befragungsergebnisse } \\
\text { (Fragen aus 6th EWCS) }\end{array}$ \\
\hline tägliche Arbeitszeit & max. 10 Std. & $\begin{array}{l}\text { 10 Std. werden häufig überschritten } \\
\text { - bei allen betrachteten Beschäf- } \\
\text { tigten zwischen } 7 \text { und 17-mal } \\
\text { im betrachteten Zeitraum } \\
\text { z.T. wochenweise gehäuft }\end{array}$ & $\begin{array}{l}\text { Wie häufig arbeiten Sie im } \\
\text { Monat mehr als } 10 \text { Stun- } \\
\text { den? } \\
\text { - nie: } 3 \\
\text { - 1-5-mal: } 13 \\
\text { - 6-10-mal: } 2\end{array}$ \\
\hline Pausen & $\begin{array}{l}\text { ab } 6 \text { Std. AZ } 30 \\
\text { Minuten; } \\
\text { ab } 9 \text { Std. AZ } 45 \\
\text { Minuten }\end{array}$ & $\begin{array}{l}\text { Pausenzeiten werden teilweise unter- } \\
\text { schritten (auch bei sehr langen tägli- } \\
\text { chen Arbeitszeiten) } \\
\text { - bei allen betrachteten Beschäf- } \\
\text { tigten 2-14-mal im betrachte- } \\
\text { ten Zeitraum } \\
\text { es ergeben sich aber auch Pausenzeiten, } \\
\text { wenn keine erforderlich sind }\end{array}$ & Nicht erhoben \\
\hline $\begin{array}{l}\text { wöchentliche Ar- } \\
\text { beitszeit }\end{array}$ & $\begin{array}{l}\text { max. } 60 \text { Std. mit } \\
\text { Ausgleich auf } \\
\text { durchschn. } 48 \text { Std. }\end{array}$ & $\begin{array}{l}\text { Ausgleich auf } 48 \text { Stunden gegeben, } \\
60 \text { Stunden werden nicht überschritten, } \\
\text { Wochenarbeitszeiten allerdings sehr } \\
\text { unregelmäßig, da sich hohe Arbeitszei- } \\
\text { ten und Ausgleich abwechseln } \\
\text { - bei } 6 \text { Beschäftigten 2-6-mal im } \\
\text { betrachteten Zeitraum }\end{array}$ & rhoben \\
\hline tägliche Ruhezeit & min. 11 Std. & $\begin{array}{l}\text { Ruhezeiten werden teilweise unter- } \\
\text { schritten } \\
\quad \text { - bei } 5 \text { Beschäftigten 1-4-mal im } \\
\quad \text { betrachteten Zeitraum } \\
\text { zumeist nur geringfügig, vereinzelt auch } \\
\text { erheblich }\end{array}$ & $\begin{array}{l}\text { Ist es bei Ihnen im letzten } \\
\text { Monat mindestens einmal } \\
\text { vorgekommen, dass weni- } \\
\text { ger als } 11 \text { Stunden zwi- } \\
\text { schen dem Ende eines } \\
\text { Arbeitstages und dem } \\
\text { Start des nächsten Arbeits- } \\
\text { tages lagen? } \\
\text { - nein: } 4 \\
\text { - ja: } 13\end{array}$ \\
\hline $\begin{array}{l}\text { Wochenruhezeit } \\
\text { und } \\
\text { Wochenendarbeit }\end{array}$ & $\begin{array}{l}\text { min. } 35 \text { Std. (inkl. } \\
24 \text { Std. Sonntag) }\end{array}$ & $\begin{array}{l}\text { Wochenruhezeiten werden eingehalten, } \\
\text { da Samstag in der Regel frei ist, } \\
\text { einige Wochenendeinsätze am Samstag } \\
\text { und/ oder Sonntag mit Ersatzruhetagen }\end{array}$ & $\begin{array}{l}\text { Wie häufig arbeiten Sie im } \\
\text { Monat am Wochenende? } \\
\text { - nie: } 8 \\
\text { - 1-2-mal: } 10\end{array}$ \\
\hline
\end{tabular}

Wochenendarbeit, insbesondere Arbeit am Sonntag, gehört für die mobil Beschäftigten meist zum Arbeitsalltag. Immerhin geben 10 Beschäftigte an, innerhalb eines Monats 1-2-mal am 
Wochenende zu arbeiten. Auch wenn hier der gesetzliche Rahmen eingehalten wird, da dieser einen großen zeitlichen Spielraum im Hinblick auf Wochenarbeitszeiten ermöglicht, sind die Belastungen durch die hohen Arbeitszeiten bemerkenswert. Sie können massiert über mehrere Wochen auftreten, weshalb sie sich kumulieren können, denn hohe Wochenarbeitszeiten gehen meist mit reduzierten Erholungszeiten einher.

Gleichzeitig sind Pausen in dem vorliegenden Beispiel, die zur Unterbrechung der Belastungen der täglichen Arbeitszeit gedacht sind, nicht selten zu gering oder entfallen, teilweise gerade dann, wenn lange am Stück gearbeitet wird. Die analysierten Arbeitszeitaufzeichnungen zeigen, dass - bezogen auf die gesetzlichen Vorgaben - Pausen bei allen 8 betrachteten Beschäftigten in den 4 Monaten 2-14-mal zu kurz sind.

Die nachfolgende Tabelle 3 stellt einen Ausschnitt der tatsächlichen Arbeitszeiten über drei Wochen eines Beschäftigten dar. Dabei sind Beginn und Ende der Arbeitszeiten zu erkennen. Am „02 Fr“ und am „15 Do“ sind jeweils 2 Einbuchungen am Tag erfolgt, die dem Original entsprechend übernommen wurden. Berechnet wurden die gesamten Anwesenheitszeiten, die Arbeitszeit nach Abzug der eingebuchten Pausen, die Pausen selbst, die Wochenarbeitszeiten sowie die täglichen Ruhezeiten. Da Samstage in dem betrachteten Zeitraum arbeitsfrei sind, sind diese hier nicht aufgeführt.

Bei dieser Detailbetrachtung zeigt sich, wie auch schon in der Übersicht der Arbeitszeiten in Tabelle 2, dass die tägliche Arbeitszeit häufig zu lang ist gegenüber den gesetzlichen Maximalvorgaben (9-mal über $10 \mathrm{Std}$.), wenn auch z.T. nur geringfügig. Damit einhergehend ist die Wochenarbeitszeit sehr hoch (2-mal über 48 Std.) und stark schwankend. Die täglichen Ruhezeiten liegen an drei Tagen unter den vorgeschriebenen 11 Stunden. Selbst wenn die verkürzten Ruhezeiten vom Beschäftigten frei gewählt sind, wie sich aus Gesprächen ergab, stellt auch dies einen Gesetzesverstoß dar. Die Pausen weisen diverse Verstöße gegen das Arbeitszeitgesetz durch Verkürzungen auf (5-mal). Durch das Fehlen oder die Reduzierung von Pausenzeiten ist eine ausreichende Erholung während der Arbeitszeit zur Vorbeugung von Belastungen nicht in ausreichendem Maße gegeben.

Aus dieser Übersicht der tatsächlich geleisteten Arbeitszeiten ergibt sich ein ähnliches Bild, wie es bereits in den Interviews mit ÜbernachterInnen deutlich wurde. 
Tabelle 3: Arbeitszeiten über 3 Wochen eines mobil Beschäftigten (Unternehmen 2)

\begin{tabular}{|c|c|c|c|c|c|c|c|}
\hline & & & & & & & \\
\hline $\operatorname{Tag}(\mathrm{KW})$ & Beginn & Ende & Anwesenheit & Arbeitszeit & Pause & $\begin{array}{l}\text { Wochen- } \\
\text { arbeitszeit }\end{array}$ & $\begin{array}{l}\text { tägliche } \\
\text { Ruhezeit }\end{array}$ \\
\hline & Uhrzeit & Uhrzeit & Std. & Std. & Std. & Std. & Std. \\
\hline 29 Мо (22) & $07: 40$ & 18:53 & 11,22 & 10,47 & 0,75 & & 12,82 \\
\hline $30 \mathrm{Di}$ & 07:42 & 18:27 & 10,75 & 10,00 & 0,75 & & 13,27 \\
\hline $31 \mathrm{Mi}$ & 07:43 & 18:34 & 10,85 & 10,10 & 0,75 & & 13,15 \\
\hline 01 Do & 07:43 & 19:03 & 11,33 & 11,33 & 0,00 & & 11,82 \\
\hline \multirow[t]{2}{*}{$02 \mathrm{Fr}$} & 06:52 & 09:24 & 2,53 & 2,53 & 0,00 & & \\
\hline & 10:03 & 14:13 & 4,17 & 4,17 & 0,00 & & \\
\hline 04 So & & & & & & 48,60 & \\
\hline \multicolumn{8}{|l|}{$05 \mathrm{Mo}(23)$} \\
\hline & $16: 20$ & $23: 40$ & 7,33 & 7,33 & 0,00 & & 8,33 \\
\hline $06 \mathrm{Di}$ & 08:00 & 19:00 & 11,00 & 10,25 & 0,75 & & 12,45 \\
\hline $07 \mathrm{Mi}$ & $07: 27$ & 12:10 & 4,72 & 4,72 & 0,00 & & 19,83 \\
\hline 08 Do & 08:00 & 19:15 & 11,25 & 10,50 & 0,75 & & 9,83 \\
\hline $09 \mathrm{Fr}$ & 05:05 & $15: 43$ & 10,63 & 10,13 & 0,50 & & \\
\hline 11 So & $14: 25$ & $21: 47$ & 7,37 & 7,37 & 0,00 & 50,30 & \\
\hline $12 \mathrm{Mo}(24)$ & 07.35 & 18.25 & 1082 ] & 1000 & 075 & & 1202 \\
\hline $13 \mathrm{Di}$ & $\frac{07.03}{07.45}$ & 18.29 & 10,83 & 10,08 & 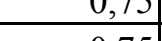 & +5 & 13,33 \\
\hline 1501 & 0/:45 & 18:5/ & 11,20 & 10,43 & 0,13 & & 12,10 \\
\hline $14 \mathrm{Mi}$ & 07:39 & 18:30 & 10,85 & 10,10 & 0,75 & & 10,50 \\
\hline \multirow[t]{2}{*}{15 Do } & 05:00 & $12: 27$ & 7,45 & 7,45 & 0,00 & & \\
\hline & 12:43 & 14:29 & 1,77 & 1,48 & 0,29 & & \\
\hline \multicolumn{8}{|l|}{$16 \mathrm{Fr}$} \\
\hline $18 \mathrm{So}$ & & & & & & 39,56 & \\
\hline
\end{tabular}

\section{Ergebnisse im Überblick und Diskussion}

Insgesamt lassen die dargestellten Ergebnisse mobil Beschäftigter sowohl für ÜbernachterInnen als auch für Nicht-ÜbernachterInnen kritisch zu betrachtende Arbeitszeitmerkmale erkennen. Als kritische Arbeitszeitmerkmale haben sich lange tägliche und wöchentliche Arbeitszeiten, ein unkalkulierbares Arbeitsende, zu kurze tägliche Ruhezeiten, zu kurze oder fehlende Pausen, Fahrtzeiten, die z.T. noch zu den Arbeitszeiten dazukommen sowie Rufbereitschaften, die nicht optimal geplant sind, herausgestellt.

Sowohl ÜbernachterInnen als auch Nicht-ÜbernachterInnen haben z.T. lange tägliche Arbeitszeiten. Diese können zur Reduzierung von täglichen Ruhezeiten und zu negativen Auswirkungen auf Gesundheit und Privatleben führen. Fehlen betriebliche Regelungen für zeitnahe Ausgleichsmöglichkeiten, was verstärkt bei ÜbernachterInnen vorgefunden wurde, erhöht sich die Wochenarbeitszeit z.T. stark und permanent.

Neben langen täglichen Arbeitszeiten ist insbesondere bei den Nicht-ÜbernachterInnen ein späteres und ungeplantes Arbeitsende genannt worden. Hierdurch können Planungen im privaten Bereich hinfällig werden. Bei ÜbernachterInnen fällt dieser Punkt weniger auf, da sie 
zu den Zeiten, in denen sie mobil unterwegs sind, kaum Zeiten mit Familie, Freunden oder sonstigen Freizeitaktivitäten abstimmen müssen.

Ein spätes Arbeitsende in Kombination mit langen Arbeitszeiten führt eindeutig zu einer Massierung der Arbeitszeiten. Dies kann von ÜbernachterInnen sogar als positiv gesehen werden. Die Erledigung des Arbeitsauftrages vor Ort, auch wenn dies längere tägliche Arbeitszeiten mit einem unkalkulierbar späten Arbeitsende bedeuten kann, heißt auch, dass ÜbernachterInnen zeitnaher oder auch längere Zeiten wieder zuhause verbringen können. Früher oder auch länger wieder zuhause zu sein, führt dazu, wieder am Familien- und Freizeitgeschehen teilnehmen zu können. Gerade bei längerem Unterwegs- und damit Wegsein ist diese Teilnahme an diesem Geschehen für die Beschäftigten besonders wichtig. Sie nehmen dafür aus einem Eigeninteresse heraus in Kauf, vor Ort länger tätig zu sein als gesetzlich erlaubt.

Betrachtet man die Auswertungen der Arbeitszeitaufzeichnungen aus Unternehmen 2, werden die Kombinationen von kritischen Arbeitszeitmerkmalen über mehrere Wochen bei den hier betrachteten ÜbernachterInnen deutlich. Dadurch steigt das Risiko physischer und psychischer Beeinträchtigungen. Es muss allerdings noch einmal betont werden, dass dieses Unternehmen ein Positivbeispiel darstellt. Aus Gesprächen und Erfahrungen mit anderen Unternehmen heraus ist zu vermuten, dass Arbeitszeiten mobil Beschäftigter generell weitaus häufiger Verstöße aufweisen. Umso wichtiger ist es, darauf hinweisen zu können, dass es sogar in positiven Fällen, wie dem vorliegenden, trotzdem noch zu diversen Arbeitszeitverstößen kommt.

Auch wenn Arbeitszeitaufzeichnungen von Nicht-ÜbernachterInnen im Projekt prentimo bisher nicht vorliegen, ist zu vermuten, dass diese extreme Konstellation von risikobehafteten Arbeitszeitmerkmalen hier eher nicht in dem gleichen Ausmaß vorzufinden ist. Diese Beschäftigten haben ein Interesse daran, ein weitestgehend planbares Arbeitsende zu haben. Zudem hat sich bei Nicht-ÜbernachterInnen herausgestellt, dass ein zeitnaher Ausgleich von Mehrarbeit besser möglich bzw. geregelt ist, weshalb die Wochenarbeitszeiten selten über die vertraglich geregelte Stundenzahl hinausgehen.

Längeres Wegsein von zuhause wird von den ÜbernachterInnen als schwierig beschrieben, da häusliche Arbeit, oder Sorgearbeit, die mobil Beschäftigte, wenn sie zuhause sind, übernehmen, anders organisiert werden muss. Umso mehr ist zu verstehen, dass gerade übernachtende mobil Beschäftigte ihre Arbeitszeit massieren, auch über die gesetzlichen Grenzen hinaus, um wieder schnell und länger zuhause sein zu können. Das Interesse an kurzen Abwesenheitszeiten entstammt jedoch nicht nur dem Privatleben, sondern auch der Tatsache, dass (berufliche) Arbeit aufläuft, die nicht unterwegs erledigt werden kann.

Neben den beschriebenen Arbeitszeitmerkmalen lag die Analyse der geführten Interviews auch auf Fahrtzeiten sowie Rufbereitschaften und den damit einhergehenden Belastungen.

Fahrtzeiten können die täglichen Arbeitszeiten zusätzlich verlängern und sind besonders kritisch, wenn sie durch betriebliche Regelungen, hier Vertrauensarbeitszeit mit Zielvereinbarungen, nicht in Gänze zur Arbeitszeit gerechnet werden, wie dies teilweise bei den betrachteten ÜbernachterInnen der Fall ist. In Kombination mit den zumeist schon hohen Wochenarbeitszeiten bleiben tägliche und wöchentliche Ruhezeiten unter den gesetzlichen Vorgaben und dies nicht nur in Ausnahmefällen. 
Gleichzeitig werden gerade bei Vertrauensarbeitszeit im Rahmen mobiler Arbeit teilweise keine Arbeitszeitaufzeichnungen durchgeführt, auch wenn dies nach dem Arbeitszeitgesetz nicht korrekt ist, denn zumindest muss eine Arbeitsdauer, wenn 8 Stunden überschritten werden, dokumentiert und 2 Jahre aufbewahrt werden ( $\$ 16$ des ArbZG).

Rufbereitschaften werden als Möglichkeit eingesetzt, die Arbeitszeiten zu flexibilisieren. Sie stellen grundsätzlich eine besondere Belastung dar, die sich aus dem Bereithalten zur Arbeit ergibt. Gesetzlich betrachtet, bedeutet das Bereithalten keine Arbeitszeit, erst mit dem Aktivwerden beginnt diese. Dafür sind andere gesetzliche Aspekte zu berücksichtigen. Ein Überblick über die Einhaltung dieser gesetzlichen Vorgaben ist zu erhalten, wenn die Inanspruchnahme von Rufbereitschaften analysiert wird (u.a. wie häufig müssen die Beschäftigten während dieser Zeit arbeiten? Wieviel Zeit nimmt diese Arbeit pro Einsatz in Anspruch? Wie lang sind die sich anschließenden Ruhezeiten?). Hieran lässt sich auch ableiten, ob Rufbereitschaften die (gesetzlich) geeignete Form ist, Arbeitszeitschwankungen aufzufangen. So ist bei einer Inanspruchnahme spätestens ab $10 \%$ anzudenken, Bereitschaftsdienste einzurichten oder ggf. auch Schichtarbeit einzuführen. Rufbereitschaften sind also grundsätzlich zu analysieren und ggf. die Arbeitszeit neu zu gestalten, um negative Auswirkungen zu minimieren. Die insgesamt vorgefundenen Auffälligkeiten hinsichtlich Arbeitszeiten sowie Fahrtzeiten und Rufbereitschaften können durch verschiedene Aspekte verursacht werden.

Grundsätzlich sollte bei der hier betrachteten Form mobiler Arbeit die Personalbedarfsplanung überdacht werden, denn die Vielzahl von Arbeitszeitverstößen lässt vermuten, dass mit zu wenig Personal geplant wird.

Zudem ist anzuraten, dass die tatsächlichen Arbeitszeiten incl. Fahrtzeiten und Rufbereitschaften in den Unternehmen mit mobil Beschäftigten erhoben und analysiert werden im Hinblick auf Verstöße gegen das Arbeitszeitgesetz. Dies gilt auch dann, wenn Beschäftigte aus scheinbar freien Stücken diese Verstöße begangen haben. Die Verpflichtung der Einhaltung, solange das Arbeitszeitgesetz in dieser Form gültig ist, liegt auf Seiten des Arbeitgebers und kann nicht uneingeschränkt auf die Beschäftigten übertragen werden.

Auch die generelle Planung von Arbeitseinsätzen ist zu überdenken. Hierzu gehören neben der für die Erledigung des Arbeitsauftrages benötigte Zeit auch Fahrtzeiten mit Berücksichtigung von tageszeitlichen Staus oder der Zeit für eine Parkplatzsuche. Dies gilt vorrangig für die Nicht-ÜbernachterInnen, denn diese leisten pro Tag meist mehrere dienstliche Fahrten, um von Kunde zu Kunde zu gelangen. So können Stressfaktoren für die Beschäftigten so gering wie möglich gehalten werden. Doch auch bei ÜbernachterInnen sind die anfallenden Fahrtzeiten ein wesentlicher Punkt, den es gilt, in die zeitliche Planung einzubeziehen. Gerade wenn sich nach einer langen Fahrtzeit ohne Unterbrechung zudem lange Arbeitszeiten beim Kunden vor Ort anschließen, kommt es zu einer Massierung von Arbeitszeit (bzw. arbeitsgebundenen Zeit, falls Fahrtzeiten nicht zur Arbeitszeit gerechnet werden), wodurch sich Belastungen verstärken. Dies gilt besonders, wenn nicht zeitnah ausreichende Ruhezeiten ermöglicht werden.

Aber mobil Beschäftigte selbst wie auch Unternehmen sehen das Arbeitszeitgesetz teilweise als hinderlich und ungeeignet an für diese mobile und damit zeitlich flexible Form der Arbeit. Das Arbeitszeitgesetz allerdings ist ein Arbeitsschutzgesetz und soll Beschäftigte vor negativen Folgen durch ungünstige Gestaltung von Arbeitszeit schützen. Im Rahmen mobiler 
Arbeit wird die Verantwortung zur Einhaltung der gesetzlichen Vorgaben an die Beschäftigten selbst abgegeben. Gleichzeitig sollen sie die anfallende Arbeit bei den Kunden erledigen, z.T. ihre Fahrten selber organisieren und zudem noch ihr Privatleben damit koordinieren. Gesetzliche Arbeitszeitvorgaben, deren Sinnhaftigkeit ohne Erläuterung der Vorteile für die Beschäftigten, auch im Sinne interessierter Selbstgefährdung, können unter diesen Bedingungen ggf. von diesen nicht ausreichend nachvollzogen werden.

Die negativen Auswirkungen der beschriebenen Arbeitszeitverstöße sind den Beschäftigten selbst, aber auch den Unternehmen selten bewusst. Gerade bei mobiler Arbeit besteht die Notwendigkeit, Gesundheits- und Gestaltungskompetenzen zu vermitteln. Diese Kompetenzentwicklung gehört zur Fürsorgepflicht der Arbeitgeber und kann in betrieblichen Leitlinien oder auch in einer Betriebsvereinbarung zu mobiler Arbeit berücksichtigt werden.

Es ist also kein einfaches Unterfangen, die Bedeutung zur Gesunderhaltung durch die Regelungen des Arbeitszeitgesetzes in die Unternehmen zu tragen. Verständnis zu schaffen und betriebsinterne Lösungen zu entwickeln, geht nur mit Beteiligung aller, d.h. mit Unternehmens- und Führungsebene, der Interessenvertretung und den Beschäftigten selbst. Hier geht es einerseits darum, zu informieren und zu diskutieren, warum z.B. die tägliche Arbeitszeit durchschnittlich nicht über 8 Stunden hinaus gehen soll oder warum Pausen eine sinnvolle Unterbrechung von Arbeitsbelastungssituationen darstellen. Andererseits gilt es auch, bisherige Arbeitsabläufe, -inhalte oder auch die Qualifikation von Personal zu hinterfragen und ggf. zu ändern, um Belastungen für mobil Beschäftigte zu reduzieren. Ein wesentliches Ziel sollte es sein, auf der Basis von Gefährdungsbeurteilungen gezielt für mobil Beschäftigte, unter Berücksichtigung gesetzlich vorgegebener Arbeitszeitmerkmale sowie Fahrtzeiten, kritische Gestaltungselemente zu erkennen, zu analysieren und betriebsintern gemeinschaftlich nach Lösungen zu suchen.

\section{Literatur}

Arbeitszeitgesetz, 24.12.2003, BGBI, I p. 1170, 1171. EU-Richtlinie 93/104/EG 23.11.1993, Abl. EG No. L 307, 18.

Beermann, Beate, Monischa Amlinger-Chatterjee, Frank Brenscheidt, Susanne Gerstenberg, Michael Niehaus und Anne M. Wöhrmann (2017). Orts- und zeitflexibles Arbeiten: Gesundheitliche Chancen und Risiken. Unter Mitarbeit von Dr. Beate Beermann. Dortmund/Berlin/Dresden: Bundesanstalt für Arbeitsschutz und Arbeitsmedizin (BAuA).

Böker, Karl-Hermann und Ute Demuth (2015). Rufbereitschaft. Düsseldorf: Setzkasten GmbH.

Breisig, Thomas, Hiltraud Grzech-Sukalo und Gerlinde Vogl (2017). Mobile Arbeit gesund gestalten Trendergebnisse aus dem Forschungsprojekt prentimo - präventionsorientierte Gestaltung mobiler Arbeit. Hg. v. prentimo. Zugriff am 15.05.2018 auf www.prentimo.de

Dettmers, Jan, Tim Vahle-Hinz, Niklas Friedrich, Monika Keller, Anika Schulz und Eva Bamberg (2012). Entgrenzung der täglichen Arbeitszeit -Beeinträchtigung durch ständige Erreichbarkeit bei Rufbereitschaft. In: Bernhard Badura, Antie Ducki, Helmut Schröder, Joachim Klose und Markus Meyer (Hg.). Fehlzeiten-Report 2012. Berlin, Heidelberg: Springer-Verlag, 53-60. 
Deutscher Bundestag (2017). Telearbeit und Mobiles Arbeiten. Voraussetzungen, Merkmale und rechtliche Rahmenbedingungen. Hg. v. Deutscher Bundestag. Wissenschaftliche Dienste. Berlin (WD 6 - 3000 - 149/16).

Deutsche Gesetzliche Unfallversicherung (2012). DGUV-Report 1/2012, Schichtarbeit - Rechtslage, gesundheitliche Risiken und Präventionsmöglichkeiten. Zugriff am 15.05.2018 auf https://publikationen.dguv.de/dguv/pdf/10002/iag-schicht-1.2012.pdf.

Ducki, Antje und Huu Tan Nguyen (2016). Psychische Gesundheit in der Arbeitswelt. Mobilität. baua: Bundesanstalt für Arbeitsschutz und Arbeitsmedizin. Dortmund/Berlin/Dresden: baua. Zugriff am 15.05.2018 auf www.baua.de/DE/Angebote/Publikationen/Berichte/F23533d.pdf? blob=publicationFile\&v $=6$.

empirica (2000). Benchmarking Progress on New Ways of Working and New Forms of Business across Europe. Brüssel: empirica. Zugriff am 15.05.2018 auf https://web.fhnw.ch/personenseiten/najib.harabi/publications/books/benchmarking-progress-oftelework-and-electronic-commerce-in-europe.

Eurofound, 6th EWCS (2015). Germany translation. Zugriff am 27.08.2018 auf www.eurofound.europa.eu/de/data/european-working-conditions-survey.

Gerstenberg, Susanne, Antje Ducki und Eva Aich (2017). Mobile Arbeit und Pendeln. In: Romahn, Regine (Hg.). Arbeitszeit gestalten. Marburg: Metropolis-Verlag, 189-202.

Grzech-Sukalo, Hiltraud (2017). Arbeitszeit als möglicher Belastungsfaktor. In: Breisig, Thomas, Hiltraud Grzech-Sukalo und Gerlinde Vogl (Hg.). Mobile Arbeit gesund gestalten - Trendergebnisse aus dem Forschungsprojekt prentimo - präventionsorientierte Gestaltung mobiler Arbeit. prentimo, 17-20.

Grzech-Sukalo, Hiltraud, Gerlinde Vogl und Thomas Breisig (2017). Mobile Arbeit: Arbeitszeit als möglicher Belastungsfaktor. Gesellschaft für Arbeitswissenschaft e.V., Frühjahrskongress in Brugg: Soziotechnische Gestaltung des digitalen Wandels - kreativ, innovativ, sinnhaft - Beitrag D.2.4.

Hupfeld, Jens, Sören Brodersen und Regina Herdegen (2013). Arbeitsbedingte räumliche Mobilität und Gesundheit. IGA-Report 25. Essen.

Janßen, Daniela und Friedhelm Nachreiner (2004). Flexible Arbeitszeiten (Schriftenreihe der BAuA, $\mathrm{Fb}$ 1025). Bremerhaven: Verlag für neue Wissenschaft.

Kaufmann, Jean-Claude (1999). Das verstehende Interview. Theorie und Praxis. Konstanz: UVK Verlagsgesellschaft.

Kraus, Sylvia und Kerstin Rieder (2018). Arbeitsbedingungen bei mobiler Arbeit - Zusammenhänge mit psychischer Gesundheit. In: Dortmund: GfA (Hg.). Frühjahrskongress 2018, Beitrag A.9.6. Frankfurt a. M.

Menz, Wolfgang und Nick Kratzer (2015). Qualitative Methoden in der Belastungsanalyse. In: Nick Kratzer, Wolfgang Menz und Barbara Pangert (Hg.). Work-Life-Balance - eine Frage der Leistungspolitik: Analysen und Gestaltungsansätze. Wiesbaden: Springer-VS, 337-353.

Nachreiner, Friedhelm (2017). Dauer der Arbeitszeit. In: Romahn, Regine (Hg.). Arbeitszeit gestalten. Marburg: Metropolis-Verlag, 105-120.

Nies, Sarah, Katrin Roller und Gerlinde Vogl (2017). Mobilität und Leistung. Dienstreisende im Trade-off zwischen Verdichtung und Entgrenzung. Arbeit 26 (2), 173-191. 
Pangert, Barbara, Nina Pauls und Heinz Schüpbach (Hg.) (2016). Die Auswirkungen arbeitsbezogener erweiterter Erreichbarkeit auf Life-Domain-Balance und Gesundheit. Forschung Projekt F 2353. Bundesanstalt für Arbeitsschutz und Arbeitsmedizin. 2., vollständig überarbeitete und ergänzte Auflage. Dortmund, Berlin, Dresden: baua Bundesanstalt für Arbeitsschutz und Arbeitsmedizin (baua). Zugriff am 05.10.2018 auf: https://www.baua.de/DE/Angebote/Publikationen /Berichte/Gd76.pdf? blob=publicationFile\&v=3.

Pangert, Barbara und Heinz Schüpbach (2014). Auswirkungen arbeitsbezogener erweiterter Erreichbarkeit auf Gesundheit und Life-Domain-Balance von Beschäftigten. Wirtschaftspsychologie (4), 73-82.

Piele, Christian und Alexander Piele (2017). Mobile Arbeit. Der Analyse des verarbeitenden Gewerbes auf Basis der IG Metall Beschäftigtenbefragung 2017. Fraunhofer IAO.

Schneider, Norbert F., Heiko Rüger und Sylvia Ruppenthal (2016). Mobilität und mobile Lebensformen. In: Handbuch Bevölkerungssoziologie. Wiesbaden: Springer VS, 501-525.

Vogl, Gerlinde und Gerd Nies (2013). Mobile Arbeit. Reihe Betriebs- und Dienstvereinbarungen, Hans-Böckler-Stiftung. Frankfurt: Bund Verlag.

Vogl, Gerlinde, Katrin Roller, Veronika Eichmann, Barbara Pangert und Nina Schiml (2014). Mobilität „rund um die Arbeit“. Ergebnisse der quantitativen Befragung. Zugriff am 15.08.2018 auf www.psychologie.uni-freiburg.de/abteilungen/wirtschaftspsychologie/personen/copy of apw/files /broschuere final 20140909.pdf.

Wirtz, Anna (2010). Gesundheitliche und soziale Auswirkungen langer Arbeitszeiten. Dortmund: Bundesanstalt für Arbeitsschutz und Arbeitsmedizin. 